\title{
EDICIONES CARTONERAS LATINOAMERICANAS EN TIEMPOS DE TRANSPOSICIÓN A DIGITAL
}

\author{
Adrián R. Vila \\ Universidad de Buenos Aires; ISFD No 6 - Chivilcoy; Universidad de Salamanca \\ editorvila@gmail.com
}

\section{RESUMEN / ABSTRACT}

El paper describe la historia de las editoriales cartoneras (EC) latinoamericanas, su evolución y la imbricación de su propio proceso productivo con formas relacionadas con la llamada "economía social" (ES) en el marco de un proceso de globalización del mercado editorial (proceso representado como GILT + ES). Se analiza la interrelación entre edición tradicional, edición cartonera y transposición a digital de dichas ediciones. Asimismo, propone una lectura de la acción de la influencia del giro poscolonial al colocar la dispositividad material cartonera como una de las formas de dicho giro, esta vez, ampliada a las formas tipográficas, ampliando los planos de acción poscolonial en el campo editorial.

PALABRAS ClAVE: editoriales cartoneras, transposición de literatura, literatura latinoamericana y caribeña, ecosistema digital.

This paper describes the history of the Latin American cardboard publishers-editoriales cartoneras (EC), their evolution and the interweaving of their own production process with practices related with the so called 'social economy'(SE), framed by the globalizing process of the publishing market (process represented as GILT $+S E$ ). The relationship between traditional publishing and cardboard publishing is analyzed, as well as their transformation to digital format. Moreover, it argues that the influence of the postcolonial turn can be appreciated in the material availability of cardboard, extended, this time, towards typographic forms, thus widening the scope of postcolonial action in the publishing field.

KEYWORDS: cardboard publishers, literary transposition, Latin American and Caribbean literature, digital ecosystem. 


\section{A Paloma Celis Carbajal, Olga Sotomayor y Nehuén Prados} por sus paciencias, aportes teóricos, pasión.

\section{INTRODUCCIÓN}

A lo largo de la historia, los libros y el contenido que portan se han distribuido como un bien escaso y como fuente de adquisición de conocimiento y entretenimiento de las élites. Al acoplarse en la actualidad los libros de manera versátil a los flujos mundiales de contenidos digitales, de manera sencilla de acceso, en cualquier lugar y momento, esto permitiría un cambio notable en la distribución de entrenamientos educativos/culturales (Wischenbart et al. 153). En un contexto de nuevas formas de escritura, de nuevas ideas de lo que es una obra y de lo que es autoría, las respuestas a los interrogantes acerca de cómo se construye la oferta editorial latinoamericana y caribeña quedan en manos de nuevos agentes y de nuevas formas de legitimación: de las formas más concentradas de la industria y de las estrategias industriales más diversificadas a la configuración de mercados editoriales más ligados a lo que se categoriza como "economía social" (ES).

Como marcan Cordón García y García-Figuerola la economía de la edición "padece la implantación definitiva de un mercado regido por los procesos de GILT (globalización, internacionalización, localización y traducción)" (Cordón García y García-Figuerola 12). Aunque destacamos en este punto que las formas materiales mediante las cuales accedemos a las literaturas latinoamericanas poseen una mayor diversificación y amplitud de estilos, de lo editorial tipográfico y de las formas de concebir la escritura, más amplias y diversas que antes de la irrupción del fenómeno digital.

Si aceptamos la idea de literatura como "mecanismo autoorganizado"(Lotman Semiófera II 28), Mignolo propone esta forma de pensar la literatura como práctica discursiva regional. Al subrayar "la necesidad de incluir la literatura no occidental, del tercer mundo, de mujeres, etc., en el canon refleja [...] la existencia de un grupo de investigadores que sienten, a nivel vocacional, que el canon debería transformarse para representar a una población plural como la americana" (Mignolo 261). La noción de descolonización de la literatura latinoamericana propone la puesta en escena de acciones de recuperación de los cánones literarios propios de las comunidades amerindias, que la acción de canonización basada en "la lengua y en los valores de las culturas colonizadoras más importantes (española y portuguesa)” logró silenciar, 
pero no suprimir (Mignolo 241). Además, la incorporación de las literaturas precolombinas al corpus de la literatura latinoamericana y caribeña expresa las maneras de la resistencia indígena ante la imposición colonial y una doble articulación frente a la lengua metropolitana: la recuperación de la propia historia de los pueblos originarios mediante la utilización del alfabeto latino (De la Garza). Si el canon latinoamericano se fue construyendo sobre la base de un lenguaje estándar y de criterios estéticos imbricados en la propia noción de "poesía" y "literatura" del colonizador (Mignolo 268) el giro poscolonial implica una estrategia de derrumbe de la hegemonía impuesta por las lenguas metropolitanas y la integración de los imaginarios que las políticas coloniales omitieron y prohibieron. A su vez, el nuevo paradigma -en línea directa con postulados estructuralistas y posestructuralistas-, disolvió las nociones modernas de autor, creación y obra de arte que precipitó el escenario de la posautonomía: fue el fin de una era en que la literatura tuvo "una lógica interna" y un poder crucial. El poder de definirse y ser regida "por sus propias leyes", con instituciones propias (crítica, academias) que debatían públicamente su función, su valor y su sentido. Debatían, también, la relación de la literatura [o el arte] con las otras esferas: la política, la economía, y también su relación con la realidad histórica. Autonomía, para la literatura, fue especificidad y autorreferencialidad, y el poder de nombrarse y referirse a sí misma. Y también un modo de leerse y de cambiarse a sí misma" (Ludmer 41). Según Diana Klinger, la literatura posautónoma implica "não somente a incorporação de materiais não ficcionais na narrativa mas, sobretudo, a idéia de que a obra não se sustenta por si só. A obra não pode mais ser lida fora do contexto cultural no qual o autor produz sua intervenção e fabrica a sua figura" (Klinger 14). El autor incorpora cierta actitud etnográfica, un lenguaje de periferia y referencias culturales consideradas 'bajas'. Esas referencias no son 'citadas' como el habla de los personajes sino que se toman como principios narrativos para poder escribir "desde adentro" de esas comunidades culturales (Klinger 14). En conexión con esta exigencia de la literatura posautónoma es que debemos leer la dispositividad material de los libros de las editoriales cartoneras (EC) latinoamericanas. El aporte de autores y obras de las EC permite rastrear un estado del arte de la imbricación de dos perspectivas fundamentales para el análisis: significaciones simbólicas y formas materiales (Chartier, Inscribir 10). Por un lado, una perspectiva literaria latinoamericana poscolonial, y por el otro, el dispositivo material mediante el cual las literaturas latinoamericanas circulan. Es decir, del giro poscolonial en el arte no solo se proyectan relatos del Sur global, también se proyecta dispositividad material, dispositivos 
del objeto tipográfico. A su vez, a los procesos de GILT mencionados, la dispositividad material cartonera le sumaría el vector de la economía social (ES), proceso que hemos descrito como GILT + ES.

\section{AHORA BIEN... ¿QUÉ SON LAS EDITORIALES CARTONERAS (EC)?}

El fenómeno de las EC fue iniciado por Eloísa Cartonera en Argentina en 2001. Esta funciona como cooperativa editorial que produce libros realizados a mano. Se inició con la idea de ofrecer respuesta a la crisis política argentina y al colapso económico de 2001 y la salida postneoliberalismo de los primeros años de la década del 2000 , cuando el desempleo forzó a miles de personas a recolectar basura en las calles de las ciudades. Muchos de esos sobrevivientes se dedicaron a seleccionar basura para el reciclaje, por lo cual pasaron a ser conocidos como cartoneros. En aquella situación de crisis social y económica, el sector cultural quedó afectado: los libros no se vendían, cerraban librerías y editoriales, los autores no podían publicar. Así, el escritor Washington Cucurto y los artistas visuales Javier Barilaro y Fernanda Laguna empezaron a confeccionar libros artísticos empleando cartón desechado. Solidarizándose con los desocupados, cambiaron el objetivo que tenían de crear libros artísticos por el de poner la literatura al alcance de todos, vendiendo sus libros a precios asequibles y pagando a los cartoneros mejor precio que en los centros de reciclaje. Con la incorporación de algunos cartoneros al equipo de producción, se creó la cooperativa Eloísa Cartonera. Esta, poco a poco, se fue constituyendo en un faro de atracción para escritores latinoamericanos que donaron sus obras para la construcción de un catálogo que se componía de relatos breves, cuentos sueltos, poemarios, novelas, textos de vanguardia, literatura infantil.

Así, las EC se constituyen en plataformas de distribución cultural que reelaboran las formas tradicionales de edición en soporte impreso:

- Sus proveedores son, por un lado, algunos de los miles de trabajadores que colectan papeles y cartones en las bolsas y artefactos de recolección de basura de las ciudades latinoamericanas y, por otro lado, la búsqueda del cartón se hace directamente por parte de los miembros de la editorial.

- Los títulos editados son donados por los autores. 
- Algunas de estas editoriales publican a desconocidos y nóveles autores de las periferias, posibilitando la edición de autores que tienen vedado el acceso a los circuitos editoriales mainstream.

- Con un trabajo de arte y diseños artesanal (las cubiertas son coloreadas a mano a acuarela y témpera, acrílico, o utilizando otras técnicas como collage, pegado y recorte de fotos, esténcil, sellos, etc.).

- Sin cadenas de distribución tradicional propias, la mayoría de sus títulos son distribuidos por los mismos autores, vendidos en sus locales o en ferias (aunque algunos de sus títulos son distribuidos por empresas de distribución tradicional).

- La mayoría de las EC no desarrollan registro ISBN de las obras editadas (hay casos en que sí hacen registro ISBN: Ultramarinay Meninas -de España-y Animita -de Chile-).

- Como las posibilidades de encuadernación presentan una limitación, por lo general se editan cuentos, novelas cortas, ensayos cortos y poesía. Aunque hay EC que editan libros en varios tomos.

- En la actualidad, las tiradas promedio de las EC son de número irregular: en el caso de la EC "faro", Eloísa Cartonera, las tiradas iniciales van de 1000 a 1500 ejemplares para autores como Aira, Piglia, Perlongher, Casas, Link, Umpi, Bejerman, Fogwill, Bellatin. En este caso, de autores conocidos, cada edición dura casi dos años. El catálogo de autores más jóvenes posee una tirada promedio de 400 ejemplares y se reeditan cuando se agota la primera edición. En el caso de las EC de Chile, las tiradas van de 1 a 30 ejemplares (en el caso del Taller Colectivo Anartistas), pasando por tiradas de hasta 50 ejemplares (Editorial "Cayó la Teja"). Sotomayor (2016) plantea que "las tiradas llegaron en algún momento a 100 ejemplares y hoy se mantienen en cifras menores a 50 ejemplares por título". En el caso de algunas EC mexicanas, las tiradas promedio van desde los 20 ejemplares (Milpa Cartonera), pasando por los 50 ejemplares (Mamá Dolores de Querétaro), por los 100 ejemplares (Pachuk Cartonera de Pachuca, Hidalgo; Cohuiná Cartonera), llegando a los 120 ejemplares (La Cartonera de Cuernavaca, que ha llegado a realizar ediciones de 500 ejemplares). Las EC peruanas realizan tiradas que van desde los ejemplares únicos ("libros objeto"), pasando por los 25-50 (Chacra Cartonera, Viringo Cartonero y La Ingeniosa Cartonera), hasta los 150 ejemplares (Sullawayta Cartonera, que además, realiza tiradas libres, es decir, publica de 5 en 5). En Venezuela (en el caso de Dirtsa Cartonera) en la primera edición se tiran entre 30 y 100 ejemplares. En Guatemala 
las tiradas promedio se desarrollan en un rango entre 50 y 100 ejemplares (Proyecto Editorial Los Zopilotes - están planificando editar con tiradas fijas-y Alambique). Fuera de Latinoamérica: en Francia las tiradas van de 10 a 100 ejemplares (Cosette Cartonera varía las tiradas de acuerdo a la lengua de los títulos: en francés, las ediciones son de 50 ejemplares, y en otra lengua varían entre 10 y 20 ejemplares; Kartocéros Editions, varía entre 50 y 100 ejemplares); en EE.UU., se detectan tiradas de 50 a 100 ejemplares (Cardboard House Press edita con tiradas promedio de 80 ejemplares); en España (La Verónica Cartonera de Barcelona tira en su primera edición 40 ejemplares y lleva títulos con 3 ediciones consecutivas) ${ }^{1}$.

Son EC cuyos catálogos fueron detectados de la Base de Datos de Editoriales Cartoneras de la Universidad de Wisconsin las que detallamos:

De Argentina: Eloísa Cartonera, Editorial Retazos, Ñaisandy Cartonera, Textos de Cartón; de Bolivia: Yerba Mala Cartonera, Nicotina Cartonera, Rostro Asado Cartonera; de Brasil: Dulcinéia Cartonera, Estação Catadora, La Katarina Kartonera, Severina Catadora, Sereia Ca(n)tadora; de Chile: Animita Cartonera, Canita Cartonera, Helecho de Cartonera, Olga Cartonera; de Colombia: Amapola Cartonera, Delahogado Elsombrero Cartonera, Patasola Cartonera; de Costa Rica: Cartonera Tica; de El Salvador: La Cabuda Cartonera, Pirata Cartonera; de Ecuador: Dadaif Cartonera, Matapalo Cartonera; de México: Cartonera La Cecilia, Cohuiná Cartonera, Cuxtitali Cartonera, Kodama Cartonera, La Cartonera, La Ratona Cartonera, La Rueda Cartonera, Regia Cartonera, Santa Muerte Cartonera; de Panamá: Pelo Malo Cartonera; de Paraguay: Mamacha Kartonera, MBurukujarami Kartonéra, Yiyi Jambó Cartonera; de Perú: Sarita Cartonera, Casa Katatay, Qinti Qartunira; de Puerto Rico: Atarraya Cartonera; de Uruguay: La Propia Cartonera. Estas editoriales disponen de un catálogo colectivo que incluye autores canónicos como Reinaldo Arenas, junto a escritores consagrados como Rodolfo Walsh, Diamela Eltit, Luisa Valenzuela, Haroldo de Campos, etc. Ksenija Bilbija, a su vez, destaca a los escritores bolivianos Adolfo Cárdenas y Christian Jiménez, los colombianos Andrés Caicedo, Andrés Nieva y Adriana Martínez, los argentinos Ricardo

Los datos de las tiradas promedio han sido obtenidos durante el mes de julio de 2016 mediante la colaboración de Olga Sotomayor, Paloma Celis Carbajal, Cristian Medina, Washington Cucurto y entrevistas por redes sociales a las distintas EC. 
Piña y Viridiana Pistorio, la paraguaya Maggie Torres y el mexicano Antonio Ramos Revilla, que "no habían publicado anteriormente en las editoriales tradicionales y cuyas primeras publicaciones llegaron a las manos lectoras dentro de las tapas sacadas de algún basural, pintadas con témpera a través de un esténcil" (Bilbija 105). El concepto de "clandestinidad" desarrollado por de Certeau (Cit. en Bilbija 38) recobraría "el valor literal". El trabajo de los cartoneros es "semiclandestino" como también lo es la producción de los libros (los autores no reciben dinero alguno por sus derechos de autor). A su vez, para el Estado, también sería "semiclandestina", "ya que no se pagan impuestos por las ganancias (Bilbija 112). Michel de Certeau describía las formas de la apropiación cultural de los sectores populares, sus formas del "consumo", como dotadas de "ardides, su desmoronamiento al capricho de las ocasiones, sus cacerías furtivas, su clandestinidad, su murmullo incansable, en suma una especie de invisibilidad pues no se distingue casi nada por productos propios, sino por el arte de utilizar los que le son impuestos" (de Certeau 38) frente a una producción de la industria cultural "expansionista" (de Certeau XLIII). Aunque las EC parten de un nivel de producción artesanal, sus libros pueden pasar por venta directa a los lectores o también pueden formar parte de circuitos de distribución y venta de la industria editorial tradicional. En el nivel de la producción, las EC latinoamericanas proponen una producción semiclandestina, revirtiendo la noción de industrialización serializada y estándar.

\section{CARTONERAS: GILT + ES}

El impacto del proceso globalizador posee diversos estamentos y diversas velocidades y escalas. García Canclini describe la globalización como "conjunto de procesos de homogeneización y, a la vez, de fraccionamiento articulado del mundo" (García Canclini 49). La frontera tecnológica en la que se ha constituido la "nube" (cloud computing) repercute sobre las pautas de productividad de todo el sistema económico internacional en sus diferentes planos. En el plano de la industria manufacturera, en el plano de las finanzas y en los diversos planos subsidiarios o estamentos que componen la economía y la organización del trabajo, de los estados y de las subjetividades. 
El acrónimo GILT (O’Hagan 76-77) remite a:

Globalización: el estudio de los aspectos de mercado que afectan a la comercialización en mercados internacionales de un producto. Significa colocar a disposición de todo el mundo un producto o un servicio.

Internacionalización: el proceso de diseño de productos de forma genérica para que permitan utilizarse en diversos idiomas y adecuarse a las características culturales "sin necesidad de modificar el componente central" (O'Hagan 76-77). Cuando un producto se coloca al alcance de todos los mercados, sus productores eligen una serie de características o funciones que van a ser universales.

Localización: la adaptación de productos en función de adecuarlos a las necesidades lingüísticas y culturales del país, región o idioma donde se pretende comercializar y usar.

Traducción: la reproducción de un texto fuente a un texto meta.

En la industria editorial, las naves insignia del libro digital, las plataformas Amazon, Barnes \& Noble, Kobo, Apple y Google Books ejercen un monopolio marcado en la articulación del mercado y en la organización de la oferta editorial. Además de que los pocos actores globales líderes, Amazon, Apple, Google, Kobo y un número creciente de líderes a escala regional y local, como FNAC, Tolino, China Mobile como así Telefónica Española asociada al Grupo Planeta, o en Brasil Livraria Cultura con Kobo, están todos orientados a formar plataformas digitales para la venta de libros (y aplicaciones y servicios asociados). A su vez, en el plano empresarial editorial "tradicional", debemos destacar que cinco grandes grupos poseen centenas de editoriales: Penguin Random House Bertelsmann AG, Hachette, MacMillan, News Corp. Verlagsgruppe von Holtzbrinck (HarperCollins) y Simon \& Schuster. Y todos, en menor o mayor medida, poseen sellos que editan en soporte digital y sellos tradicionales y en digital, a la vez. Asimismo, transponen títulos de soporte papel a digital y editan títulos nacidos en digital. Demás está decir que esta es la élite mundial que, a su vez, está acompañada por empresas que operan como ellas pero a escalas regionales o más pequeñas, con un grado de penetración inferior y que funcionan, a su vez, como líderes editoriales en áreas idiomáticas distintas. A saber: Pearson, McGraw Hill, Hachette, Comcast, Mediaset, Canal +, Prisa, Reuters, Planeta, etc. En relación con la integración de los grupos que editan en castellano, los más importantes son Prisa y Planeta y la división hispana de Bertelsmann (Random House 
Mondadori). Estos grupos poseen activos en múltiples negocios dentro y fuera del conglomerado de las industrias culturales.

Bilbija destaca que las cartoneras funcionan como iniciativas antimercado en el caso de la publicación de autores noveles, de experimentación, de "vanguardia", "desconocidos para la gran mayoría de lectores y demasiado riesgosos para las inversiones de las globalizadas editoriales transnacionales, regidas por el mercado irónicamente llamado libre y determinado por la competencia y la ganancia. En este sentido, la cartonera es una iniciativa anti-mercado" (Bilbija 5).

La economía social (ES) es un sector de la economía que estaría a medio camino entre sector privado y negocios por un lado, y sector público por otro lado. Incluye a cooperativas, mutuales, empresas de trabajo asociado, organizaciones no lucrativas y asociaciones caritativas. De esta forma, la idea de economía social implica una concepción de relaciones económicas que, más que reproducir el capital, priorizan los lazos sociales sobre la acumulación de capital. Así, estas unidades económicas se resumen (Defourny 90) en las características de supremacía del trabajo sobre el capital, finalidad de servicio más que de lucro, autonomía de gestión respecto del Estado y procesos de gestión democrática, más que a la economía de mercado. En este tipo de emprendimientos ubicamos a las ediciones cartoneras latinoamericanas y caribeñas.

\section{¿TRASH? ¿RR (RITUALES DE RESISTENCIA)?}

La edición cartonera deconstruye el proceso editorial constitutivo de la galaxia Gutenberg: exhibe las formas de su producción; artesaniza gran parte de la construcción del producto; descomercializa la cadena de proveedores; desprofesionaliza arte, preprensa, comercialización; expone distintas formas productivas y/o las asocia con formas de comercialización corporativas de la mano de las redes sociales y las tecnologías digitales. Y, a su vez, opera como nuevos emprendimientos en economías de base cultural de pequeña escala, renovando y ampliando el sector de la gestión cultural: funciona, mayoritariamente, como propuesta de colectivo artístico; las editoriales se desempeñan como centros culturales donde se planifican y ejercen actividades que van desde muestras de arte e intervenciones teatrales a la edición in situ de títulos cartoneros. Si las bibliotecas actuales rotan su funcionamiento y se reorganizan como centros de entrenamiento, como "espacios versátiles, 
espacios polivalentes" y establecen dinámicas comunitarias, abiertas y participativas que estimulan la creatividad y ofrecen "oportunidades de aprendizaje en contextos informales, tales como espacios para reuniones y encuentros para el público en general, para grupos comunitarios y otras organizaciones locales" (Alonso Arévalo y Vázquez Vázquez 202), también las cartoneras funcionan como fábricas/centros culturales.

El fenómeno editorial cartonero puede ser descrito también como una versión latinoamericana de la llamada cultura trash, es decir "la trivialización utilitaria, diseñada a la medida de la sociedad de la cultura de masas, de una serie de procesos culturales complejos y con cierto pasado a sus espaldas" (Costa 149). En este punto, las ediciones cartoneras funcionarían, desde la apreciación de Costa (144) sobre el trash, como "instrumentalización del mal gusto" como fenómeno estético "tan heterodoxo como cargado de potencial liberador", aunque, más que constituirse en una forma trivial de reutilización del "mal gusto" (Costa 144), las ediciones cartoneras integran la dispositividad material de la galaxia Gutenberg, constituyéndose en artefactos culturales propios de la oferta específica de las literaturas latinoamericanas. En algunos casos los textos que ya circularon como libros o como parte de libros "tradicionales" son transformados y reacomodados "en un nuevo patrón que actualiza un nuevo significado, su traslación a un contexto nuevo y su adaptación" (Clarke 275). La perspectiva del Centro de Estudios Culturales Contemporáneos (CCCS) de Birmingham ubica este tipo de estrategia de bricolage descrita por Levi-Strauss dentro del conjunto de prácticas llamadas "rituales de resistencia" $(R R)$. Esta categoría-generalmente asociada a las formas de vestir-describe el proceso mismo en que las propias formas pueden producir nuevos significados y significados alternativos a las mercancías culturales creadas para otros mercados (Clarke 275). El concepto de bricolage describía para el mencionado Levi-Strauss (Pensamiento 35-36) el reordenamiento y recontextualización de objetos para comunicar nuevos significados. Todo en el marco de un sistema semántico que contiene significados anteriores de esos objetos utilizados. Si para Levi-Strauss el bricoleur de mitos trabajaba con objetos naturales o disponibles de forma natural, nosotros podríamos argumentar -con Clarke y el planteo de rituales de resistencia $(R R)$ de los CCCS- que uno de los vectores para el análisis de las ediciones cartoneras latinoamericanas y caribeñas es su articulación como bricolage cultural. Es decir, productos nuevos que ya cargan con significados y mensajes a partir de sus textos y su dispositividad material. En la heterogeneidad de propuestas del universo de la edición cartonera no se distinguen tópicos literarios propios 
así como tampoco se detectan formas literarias, poéticas, programas literarios específicos o "criterios de selección compartidos" (Celis Carbajal). Es más, las piezas de la galaxia Gutenberg, los textos, los autores, las formas de distribución, las formas ligadas al "estilo" y las poéticas no solo existían previamente: las ediciones cartoneras se insertan en ese sistema cambiando y transformándolos. Pero en su nueva dispositividad material sus textos pueden expresar el punto de vista de un grupo opuesto a los valores de la sociedad mayoritaria en el marco de "una oposición parcialmente negociada" a esos mismos valores (Clarke 274).

\section{UNA DISPOSITIVIDAD MATERIAL LATINOAMERICANA Y CARIBEÑA}

Para Roger Chartier es importante distinguir las estrategias de puesta en texto de las estrategias de puesta en soporte. A las consignas que el autor inscribe en la obra de acuerdo a su "intención" se le cruzan los procedimientos propios de la impresión, que "pueden sugerir lecturas diferentes de un mismo texto" (Chartier, Mundo 79). Esto es, no disociar "el análisis de las significaciones simbólicas del de las formas materiales que las transmiten" (Chartier, Inscribir 10). Cordón García acota que lo que hace que Chartier afirme "que ningún orden de los discursos es separable del orden de los libros de los que son contemporáneos es esa conciencia sobre la importancia de los protocolos de lectura presentes en la forma de cada texto..." (Cordón García, Lecturas 27). Es decir que la dispositividad material mediante la cual circulan los textos de las literaturas latinoamericanas que publican se organiza como especificidad editorial mediante la cual circulan formas literarias propias de Latinoamérica y el Caribe. Ya en la década de los 90, Stuart Hall sintetizaba que la revolución cultural más profunda ha sido una consecuencia de las márgenes entrando a la representación:

en el arte, en la pintura, en el cine, en la música, en la literatura, en las artes modernas de todos lados, en política y, en términos generales, en la totalidad de la vida social. Y estas márgenes no lo hacen para ser situadas por otro régimen, por el ojo del imperio, sino que lo hacen para reclamar para sí mismas alguna de las formas posibles de representación. En nuestro mundo, de manera paradójica, la marginalidad se ha convertido en un espacio poderoso (Hall 37). 
Esta incorporación poderosa a escala global de una corriente artística determinada por "valores locales, nacionales, anticoloniales, independientes y antiglobalización", circula hoy por los circuitos del mainstream artístico internacional. Es decir que el llamado giro poscolonial ha hecho "que se vuelva predominante en los circuitos de arte internacional" (Smith 22, 23, 331). En este tópico, tal vez se pueda establecer un paralelismo con lo que sucede en el mundo de las artes visuales. Smith señala que el llamado giro poscolonial ha hecho que el arte de la periferia "se vuelva predominante en los circuitos de arte internacional" (Smith 22, 23, 331) y, para nosotros, puede constituirse en una perspectiva de interpretación de la construcción de la oferta editorial cartonera tanto en impreso como en su transposición a soporte digital de literatura latinoamericana y caribeña a escala global.

En este punto, se torna necesario remarcar que un segmento importante de la literatura latinoamericana se incorporó a la tendencia "central" de la literatura mundial de la mano de la llamada literatura del boom de la década del 60. La corriente ligada a este boom se incorporó a la corriente principal de la literatura en castellano de manera anticipada a lo que describimos como inclusión al mainstream de las formas artísticas latinoamericanas y del Tercer Mundo con el llamado giro poscolonial. Antes del boom, la literatura latinoamericana no registraba ventas masivas y ahora ingresaba al mercado de bienes simbólicos de la mano de la masividad de sus autores, que ganaron los medios de comunicación de la época. La primera edición de Cien años de soledad de Gabriel García Márquez, en 1967, por la editorial Sudamericana, tuvo una edición total de 8.000 ejemplares; a los pocos días, otra edición de 8.000 ejemplares, luego varias reimpresiones de 100.000 ejemplares. Hasta la fecha lleva vendidos más de 30 millones de ejemplares y ha sido traducida a 35 idiomas. Cifras de este tenor expresan que algunos de los autores canónicos latinoamericanos (Julio Cortázar, Jorge Luis Borges, Gabriel García Márquez, José Donoso, Mario Vargas Llosa, Miguel Ángel Asturias, Alejo Carpentier) ya formaban parte del mainstream literario internacional.

Graw propone modificar el modelo de "autonomía relativa del arte" (Bourdieu 248) para plantear un modelo de "heteronomía relativa", es decir que "las restricciones externas prevalecen pero de manera relativa" en el mundo del arte (Graw 199-205). Las restricciones externas (económicas, de mercado) se ubican en primer plano en el campo artístico, de la mano del "predominio del sistema económico en la sociedad" y como consecuencia de constituirse en la forma de pensar predominante dentro del mundo del arte (Graw 200-201). Si en el modelo de autonomía relativa primaba la 
idea de diferenciación del arte "hacia un mundo propio", en el modelo de heteronomía relativa propuesto con cierta efectividad conceptual por Graw, los acontecimientos económicos "externos" poseen un poder que se acentúa, pero no como un absoluto, sino de manera limitada. En las ediciones cartoneras latinoamericanas, se destaca lo que Graw plantea para el mundo de las artes plásticas: los artistas buscan libertad en relación con cualquier intervención a lo percibido como "externo" al campo del arte porque, entre otras cosas, "el mercado del arte depende de la creencia de que sus productos están lejos del mercado" (Graw 196). Si la tensión en el arte moderno fue por la independencia absoluta del campo literario de lo económico, es decir, finalizar con la autonomía relativa de la literatura para pasar a la autonomía absoluta, relevamos autores de las EC que establecen como estrategia propia o bien no publicar en soporte papel o bien solo publicar en ediciones cartoneras. Y, en algunos casos, solo publicar en versiones en digital sin transposición en el sentido inverso a soporte impreso.

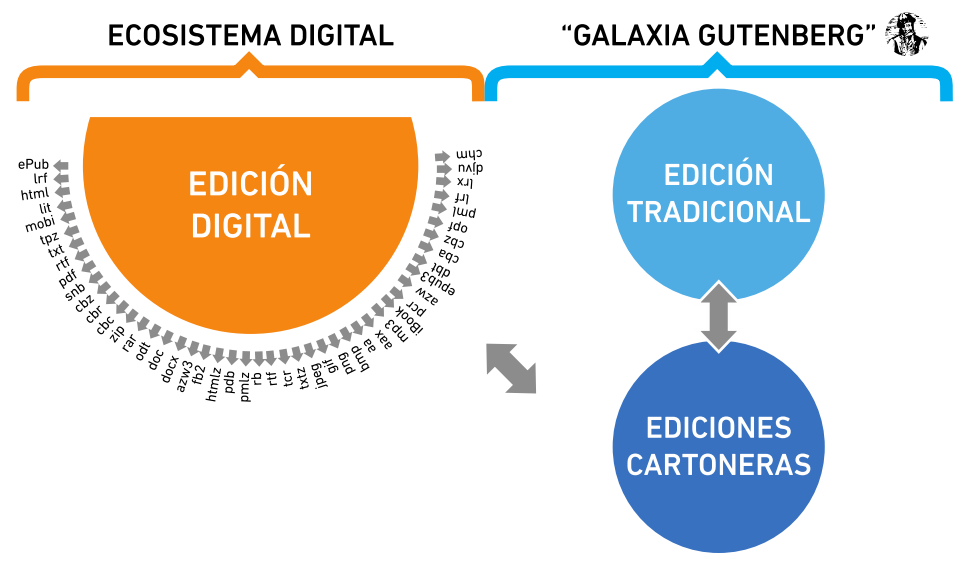

Figura 1. Esquema de dispositividades materiales: formas del ecosistema digital, galaxia Gutenberg (edición tradicional + ediciones cartoneras). Relaciones. Elaboración propia. 


\section{RELACIONES I: EDICIONES CARTONERAS-ECOSISTEMA DIGITAL}

Habida cuenta de que gran parte del catálogo general de la literatura latinoamericana y caribeña está siendo transpuesta de soporte impreso a las formas de la edición digital, las ediciones cartoneras no están exentas de ese recorrido. La incorporación de títulos de EC en plataformas de comercialización $\mathrm{y}$ webs de intercambio nos habla de un proceso en el que la noción de giro poscolonial se reconvierte. García Canclini se preguntaba (Jóvenes 28): “Es posible democratizar no solo el acceso a los bienes, sino hibridarlos, de combinar los repertorios multiculturales que expande esta época multicultural? La respuesta depende, ante todo, de respuestas políticas y económicas". La idea de procomún es como una de las respuestas a aquella pregunta de García Canclini (Jóvenes 28): destaca los compromisos sociales y negociaciones yuxtapuestas y coetáneas a la administración y funcionamiento de recursos compartidos. En este punto, debemos destacar que un porcentaje importante de títulos de EC que están disponibles en digital están bajo dominio de licencias copyleft: aquellas que permiten que cada persona que recibe una copia de una obra puede utilizarla, modificarla, redistribuirla y redistribuir las versiones derivadas de ellas. La combinación de materialidad cartonera y promoción de títulos en plataformas comercializadoras multinacionales (tanto en soporte impreso como en digital) nos describe un territorio poroso donde se combinan prácticas que podrían llegar a ser interpretadas, también, como contradictorias. Los cambios continuos en las formas de producción ligadas a la creatividad en algunos segmentos "jóvenes" de la región, las formas de apropiación del conocimiento, su utilización con un cierto valor agregado y la recirculación de las formas culturales a partir de la reutilización nos hablan también de una heterogeneidad y de hibridación en torno a las propuestas de producción y distribución de los fondos bibliográficos de las EC.

En una investigación (Vila 1-331) dedicada al estudio de la transposición a digital de un corpus de literatura latinoamericana y caribeña realizada (en el marco del Programa de Doctorado en Sociedad del Conocimiento de la Universidad de Salamanca) se destacan algunos resultados de las búsquedas en soporte digital de títulos de EC. Dichas búsquedas se realizaron en las plataformas Amazon, Barnes \& Noble, Google Books, iBookstore / iTunes, Kobo, Free-Ebooks, Casa del Libro, Gandhi, Librería Santa Fe, Livraria Cultura, Perubookstore.com, Nubico, en la web Scribd, en las bibliotecas Gallica (Francia), Columbia University Libraries, Biblioteca del Instituto Latinoamericano de Berlín, Biblioteca Nacional de España, Public Library 
New York, Europeana (Biblioteca digital de la Unión Europea), Biblioteca Nacional de Perú y en Biblioteca de São Paulo.

Partiendo de los títulos de autores latinoamericanos de que dispone la Base de Datos de Editoriales Cartoneras de Universidad de Wisconsin, se destacaron en el mencionado catálogo 487 títulos de 377 autores latinoamericanos y caribeños y se subraya que existe transposición de EC a digital. En este punto, se destaca que el proceso de transposición pudo efectuarse primero de impreso "industrial tradicional" a digital o se transpuso de impreso "industrial tradicional" a cartonera, y de ese soporte a digital de manera paralela. Las búsquedas en digital de estos títulos se realizaron en las principales plataformas de venta de ebooks y las principales bibliotecas digitales y se compararon en ellas las ofertas de dichos títulos y autores en soporte papel y en formato digital.

En los resultados de las búsquedas se destacan Google Books y la Biblioteca del Instituto de Estudios Latinoamericanos de Berlín. Google Books dispone en soporte impreso de 300 títulos de este segmento de 487 títulos en total (el $61 \%$ de los títulos hallados). Y la Biblioteca del Instituto de Estudios Latinoamericanos de Berlín es la biblioteca (además de la de Universidad de Wisconsin) que más títulos del catálogo de ediciones cartoneras dispone en impreso: 156 títulos (el 32\%) de 487 del total.

A su vez, de los resultados totales de las búsquedas en soporte digital de los títulos de autores latinoamericanos y caribeños de la Base de Datos de Editoriales Cartoneras de Universidad de Wisconsin se encuentra transpuesto a digital el 15,40\% de los títulos (75 títulos sobre 487). Destacamos que la web de intercambios Scribd ofrece 55 títulos de 36 autores (hay autores con más de un título transpuesto-), es decir, Scribd ${ }^{2}$ dispone en digital del $73,33 \%$ de los títulos transpuestos. Los mismos han sido incorporados a la mencionada web de intercambio bajo la forma de "documentos", es decir, agregados a esa web en formato digital pdf por parte de los usuarios. Esto nos

2 Scribd es una aplicación online en la que se pueden subir documentos con opciones de descarga en formato *pdf y *txt. Posee un catálogo de 700.000 libros a los que se accede por "tarifa plana" y ofrece 150.000 ebooks de la editorial Harlequin. Permite explorar, leer y compartir millones de obras: guías, manuales, informes, presentaciones y publicaciones de Random House, Harvard Press, University of Chicago y Duke Press, entre otras. Incluye: lector *epub y *pdf; documentos que pueden ser guardados para ser leídos sin conexión a Internet; funciones para compartir lecturas, etc. Y funciona como una red social en la cual se puede seguir a otros usuarios y ver su actividad. 
marca que solo una cuarta parte de los títulos en digital han sido incorporados a plataformas con fines comerciales, el restante $75 \%$ ha sido digitalizado por los usuarios. La utilización de nuevas tecnologías y, sobre todo, la puesta en digital de parte de los catálogos de las EC mediante formas de acceso abierto y/o bajo licencias copyleft, sumado a la libre disponibilidad de títulos digitalizados por los usuarios a la web Scribd, nos habla también de una doble estrategia. Por un lado, una intención de utilización de los textos de manera distinta por parte de otros y nuevos lectores, en este caso, la utilización de los textos editados primero en EC y que luego son transpuestos a digital. Si para Chartier (Mundo XX) "la transformación de las formas a través de las cuales se propone un texto legitima recepciones inéditas creando nuevos públicos y nuevos usos", el lector reutiliza el texto del autor y lo coloca a disposición de los usuarios de la web de intercambio Scribd, que en este caso, funciona como una red social. En este punto, la intervención de los usuarios en Scribd funciona como una ventana por la cual los criterios poéticos, estéticos, de "gusto" del lector ingresan en las formas de construcción del catálogo general en digital. La transposición a digital de sus propios títulos por parte de las EC también denota una estrategia de creación de nuevos públicos (y usos del mismo texto). Y otra de las combinaciones posibles en relación con la oferta de EC en plataformas: muchos de los títulos en venta en esas plataformas son de libre acceso en páginas de Facebook, blogs y páginas web de las propias editoriales. Asimismo, en esa transposición a digital persiste una actitud antimercado en la colocación en acceso abierto en digital de esos títulos propios. Es decir, productos nuevos que ya cargan con significados y mensajes a partir de sus textos y su dispositividad material. Pero en el pasaje a digital, en esa nueva dispositividad, sus textos pueden expresar el punto de vista de un grupo opuesto a los valores de la sociedad mayoritaria en la oferta de circulación abierta, de acceso libre de esos textos en el marco de "una oposición parcialmente negociada" a esos mismos valores (Clarke 274-275). Demás está decir que esos textos provenientes de EC en grandes plataformas forman parte de esa (parcial) negociación.

\section{RELACIONES II: EDICIONES CARTONERAS-ECOSISTEMADIGITAL. ANÁLISIS A PARTIR DE GRAFOS}

En matemáticas y en ciencias de la computación un grafo es un conjunto de objetos unidos por enlaces, los cuales permiten representar relaciones 
binarias entre los elementos de ese conjunto. Estas relaciones además tienen un atributo denominado peso, el cual determina el valor de dichas relaciones. El llamado grado de un objeto es la cantidad de relaciones que tiene, siendo los objetos de mayor grado quienes están vinculados con una mayor cantidad de objetos diferentes, mientras que el llamado grado con peso es la suma de los pesos de todas las relaciones de un objeto, siendo el objeto de mayor grado con peso, el que tenga relaciones mas fuertes. Desde un punto de vista práctico, los grafos permiten estudiar las interrelaciones entre objetos que interactúan unos con otros. Y permiten representar la estructura de una red, en la que los vértices (nodos) se encuentran comunicados mediante líneas. Estas líneas se denominan arcos si se observa un sentido (dirección) de la conexión y se denominan aristas si el sentido de la conexión es bidireccional (Alonso Berrocal et al. 87. Teniendo en cuenta las relaciones y sus pesos correspondientes, es matemáticamente posible agrupar los objetos en las denominadas comunidades, es decir, subconjuntos de objetos que se relacionan más entre ellos mismos que con el resto. En el caso particular de este estudio, el conjunto es el corpus de títulos de EC, los objetos del grafo son cada uno de los títulos y un enlace entre dos títulos indica que ambos aparecen en la misma plataforma en el mismo soporte. Dicho enlace tendrá peso uno, y si dos títulos aparecieran en más de una plataforma en el mismo soporte el peso de la relación aumentaría proporcionalmente. A continuación (Figuras 2 y 3 ) pueden verse los grafos correspondientes a las búsquedas en soporte impreso y digital respectivamente. Los títulos representados con el mismo color pertenecen a la misma comunidad. 


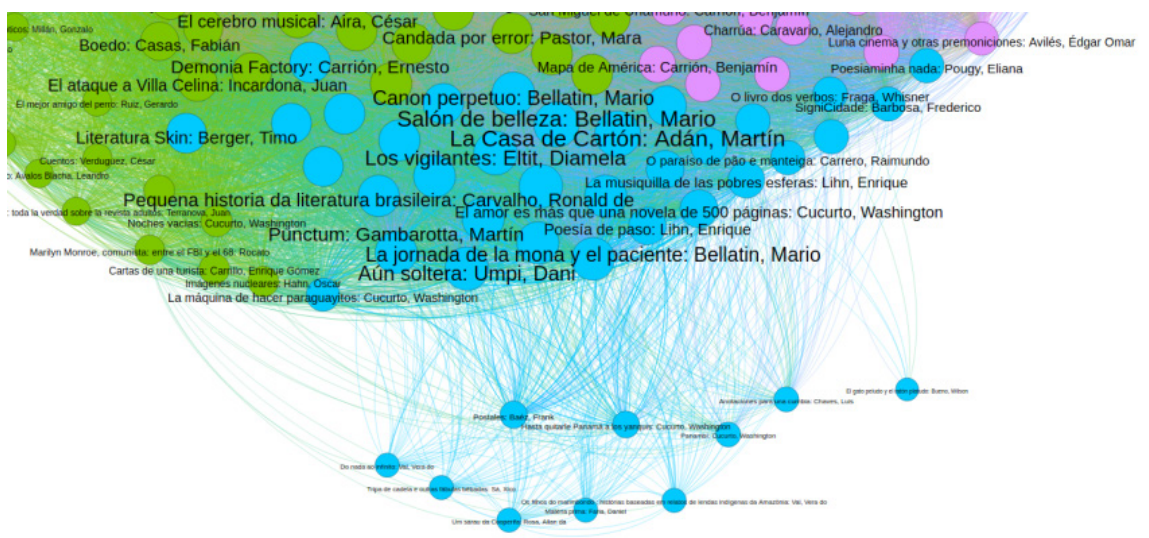

Figura 2. Recorte de los títulos y autores con mayor grado de cartoneras en impreso. Grado con aristas y etiquetas. Elaboración propia.

En el caso del grafo de los títulos de literatura latinoamericana y caribeña de la Base de Datos de Editoriales Cartoneras de Universidad de Wisconsin (hallados en soporte impreso en 21 bibliotecas y plataformas), al haber una mayor cantidad de títulos relacionados (debido a la mayor disponibilidad de títulos hallados en soporte impreso), las diferencias en el grado con peso son más sutiles. Se pueden observar solo los títulos con sus autores agrupados en 3 grandes comunidades (nodos color verde, celeste y fucsia).En una resolución a alta definición de pantalla de ordenador (V. Figuras 2 y 3 ) se observa que poseen un mayor grado con peso (en el grafo, los títulos y autores con mayor cuerpo tipográfico) los títulos Salón de belleza y La jornada de la mona y el paciente, ambos de Mario Bellatín; La casa de cartón, de Adán Martín; Punctum, de Martín Gambarotta; Aún soltera, de Dani Umpi; y Los vigilantes, de Diamela Eltit (los seis títulos y autores en la comunidad de nodos color celeste). En este punto, inferimos del grafo de Cartoneras en impreso. Grado con aristas y etiquetas que seis obras rara vez aparecen separadas y que se suelen distribuir en conjunto (en plataformas y en bibliotecas, en soporte digital). Y que de los 6 títulos con más grado, 5 de ellos son títulos editados por Eloísa Cartonera (los títulos de Mario Bellatín, Dani Umpi, Adan Martín y Martín Gambarotta). El título restante (Los vigilantes, de Diamela Eltit) fue editado por Sarita Cartonera, de Perú. Con menor grado, alejados del centro, los títulos de la comunidad color celeste: Um saráu de Cooperifa, de Alan de Rosa y El gato peludo y el gato platudo, de Wilson Bueno. Se infiere 
que estos títulos aparecen mayormente separados del resto de los títulos y que las plataformas y bibliotecas las ofrecen aleatoriamente separados, en soporte impreso.

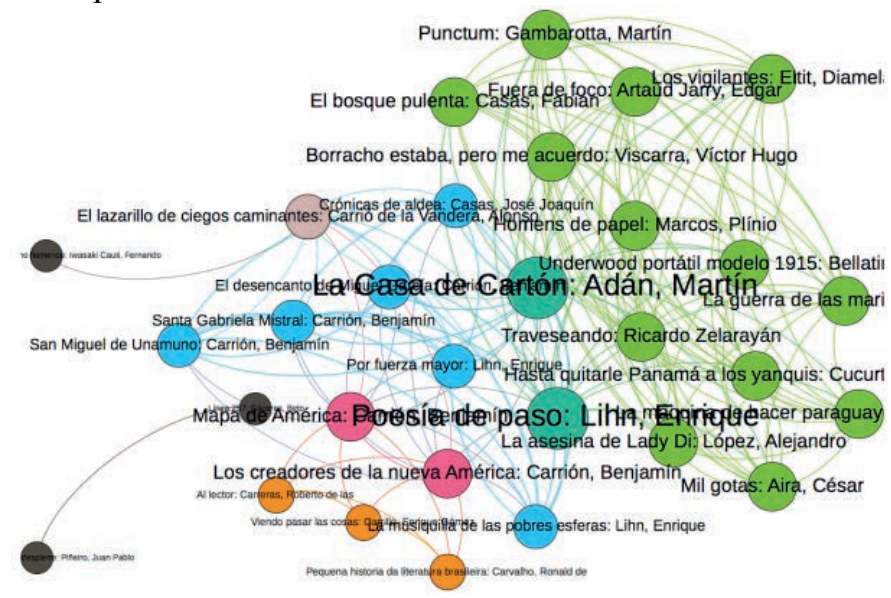

Figura 3. Grafo de cartoneras en digital. Grado con aristas y etiquetas.

Elaboración propia.

En el caso del grafo de los títulos de literatura latinoamericana y caribeña de la Base de Datos de Editoriales Cartoneras de Universidad de Wisconsin (hallados en soporte digital en 21 bibliotecas y plataformas), se puede observar cómo los títulos La casa de cartón, de Adán Martín, y Poesía de paso, de Enrique Lihn, son los que tienen un mayor grado con peso, perteneciendo estos dos a la misma comunidad de la que solo ellos forman parte, resultando estos dos títulos los más representativos del conjunto. En este punto, inferimos del grafo que estas dos obras rara vez aparecen separadas y que se suelen distribuir en conjunto (en plataformas y en bibliotecas, en soporte digital). Estos dos títulos fueron editados por Eloísa Cartonera. Dos títulos de Benjamín Carrión los siguen en grado con peso (los dos nodos en color rosa): Los creadores de la nueva América y Mapa de América (ambos en dominio público). Tres títulos poseen menor grado con peso (nodos en negro): Cuando Sara chura despierte, de Juan Pablo Piñero; Linea 257, de Beto Cáceres, y Mi poncho es un kimono flamenco, de Fernando Iwasaki. Estos tres títulos aparecen separados del resto de los títulos, ya que las plataformas y bibliotecas las ofrecen aleatoriamente separados, en soporte digital. 
Destacamos que (tanto en los resultados de los grafos obtenidos de EC en impreso como en digital) los títulos de Eloísa Cartonera funcionan como un "subcanon cartonero" cuyos títulos ocupan el espacio central en la distribución en plataformas y bibliotecas en ambos soportes. Además, en digital ocupan un espacio central títulos en dominio público como los de Benjamín Carrión, un importante escritor, político, diplomático y promotor cultural ecuatoriano, recuperado durante los últimos años por el gobierno de ese país sudamericano.

\section{CONCLUSIONES}

Terry Smith destaca que una historia del arte contemporáneo no debería escatimar esfuerzos por establecer una línea cronológica, reconocer las herencias positivas y problemáticas del arte anterior, prestar atención al arte que se produce en todo el mundo, en su espacio local y en la circulación internacional y reconocer "tal vez por primera vez, que se trata del arte del mundo (es decir, no un arte universal con distintas manifestaciones locales, tampoco artes locales dependientes de la colonización o la globalización, sino artes generadas por la diversidad del mundo)" (Smith 327-328). El arte actual, así, "está determinado de una forma más profunda por su situación dentro de la contemporaneidad", es decir, un arte en y de la contemporaneidad, pero de una contemporaneidad global que también fue permeada por el llamado giro poscolonial que, de manera pedagógica, "generó la necesidad de relatos que funcionaran en el plano global, regional y local" (Smith 21). En este punto, se puede desprender una nueva hipótesis interpretativa, construida inductivamente y por analogía con la perspectiva de Smith: del giro poscolonial en el arte no solo se proyectan relatos, también se proyecta dispositividad material, dispositivos del objeto tipográfico. Productos de la galaxia Gutenberg y de la miseria, desde este encuadre teórico las ediciones cartoneras se constituyen en dispositivos tipográficos del giro poscolonial en el marco de un proceso transnacional de reconfiguración del negocio editorial (GILT), al que amplían con la irrupción de formas de producción no tradicionales como las de la economía social (ES), resultando un proceso ampliado que se combina con el global (GILT + ES). En este punto, también, se encuadran los procesos de transposición a digital de las EC.

De la propuesta (poscolonial) de las EC a los títulos en formato AZW3 de Amazon que constituyen "lo último" del ecosistema digital existe una 
frecuencia de ofertas mayor a las disponibles hace menos de una década a nivel de los dispositivos materiales formales mediante los cuales circula y se accede a la literatura. Muchas de las obras de la literatura latinoamericana y caribeña tienen la opción de ser transpuestas a digital desde el "modo tradicional" de edición en impreso. Pero también pueden ser transpuestas a las formas de edición cartonera por fuera del "modo tradicional". En parte, la transposición a digital de títulos de edición cartonera se realiza mediante la publicación en blogs de autor o de eventos tales como los distintos $\operatorname{saraus}^{3} \mathrm{y}$ grupos de poesía de autores asociados. Y, por otro lado, la acción en el sentido inverso: los materiales nacen en aquellas formas digitales y se transponen a impreso mediante la edición cartonera en papel.

En las búsquedas realizadas en 9 bibliotecas y 14 plataformas comercializadoras y servicios de lectura en streaming destacamos que la oferta de títulos transpuestos a soporte digital de títulos de Base de Datos de Editoriales Cartoneras de Universidad de Wisconsin se encuentran el 15,40\% de los títulos (75 títulos sobre 487 del total). Y de éstos, casi el 75\% ha sido transpuesto a la web de intercambio Scribd por parte de los usuarios. Entonces, se destaca que solo una cuarta parte de los títulos en digital han sido incorporados a plataformas con fines comerciales, lo que demuestra una débil estrategia de utilización de recursos tecnológicos para la venta de títulos.

A su vez, los grafos obtenidos con la utilización de programas de software a partir de la mencionada base de datos nos permiten destacar que los títulos de la primera editorial cartonera-Eloísa Cartonera- funcionan como "subcanon cartonero": ocupan el espacio central en la distribución en plataformas y bibliotecas en ambos soportes. La posición central en los grafos de estos títulos parece ajustarse al concepto de "visibilidad consolidada" propuesto por Cordón García para aquellas obras que poseen un alto reconocimiento, entre otras razones "porque son obras que poseen un alto valor simbólico" (Cordón García, Visibilidad 17). Lotman destacaba que "en la historia de la literatura se conocen casos en que la percepción de tal o cual obra por los lectores fue determinada por la reputación de la edición en que fue publicada" (Lotman, Semiósfera I 80). El peso de la tradición generada al interior del

Los saraus se conocen actualmente como un movimiento cultural nacido en la periferia empobrecida de São Paulo. En la mayor ciudad de Brasil se vienen realizando desde 2001 reuniones en bares de diferentes barrios donde se declaman y/o leen textos propios y de terceros. La corriente surgida de este práctica se autodenomina "Movimiento de Literatura Marginal". 
movimiento por parte de Eloísa Cartonera, el fenómeno irradiador de su experiencia editorial y la calidad de los títulos editados podrían ser las bases de ese reconocimiento.

La incorporación de "formas literarias que provienen de la subalternidad" (Celis Carbajal) habilita concluir que las EC funcionan también como dispositivos materiales mediante los cuales, además de ampliar el acceso a la edición, también permiten colocar textualidades subalternas en lo socialpolítico. Si "ciertos autores que empezaron en la subalternidad han permeado los catálogos mainstream" (Celis Carbajal), también habilitan la llegada de los márgenes a la representación (Hall 37).Esa incorporación funciona en algunos casos como rituales de resistencia $(R R)$ en los que las formas mismas pueden producir nuevos (alternativos) significados (Clarke 275) al del mismo texto editado en soporte "tradicional" en el caso de transposición de ediciones tradicionales a EC. También, en muchos casos, se presentan como rituales de resistencia textos editados por primera vez en EC: la sola dispositividad material se plantea como exhibición de prácticas de oposición a las maneras tradicionales de edición y circulación de los textos. Y amplía los públicos de apropiación de las literaturas. La irrupción de voces de las periferias latinoamericanas en catálogos en los que comparten espacio con autores consagrados amplía la oferta cultural en dos vectores: literaturas de las periferias que acceden a la edición y formas de producción de dispositividad subalterna en el marco de la galaxia Gutenberg. En este punto, la irrupción de las llamadas EC constituye lo que llamamos "especificidad editorial latinoamericana". Chartier sostiene que tanto las distintas transformaciones de representación del teatro shakesperiano en EE.UU. estudiadas por Lawrence Levine, como las transformaciones "tipográficas" operadas por los editores de la Biblioteca Azul sobre las obras que incluían en su catálogo en el Antiguo Régimen francés, apuntaban a incorporar esos textos "en una matriz cultural que no es la de sus primeros destinatarios y permitir así una pluralidad de apropiaciones" (Chartier, Mundo 42). La utilización de nuevas tecnologías y, sobre todo, la puesta en digital de parte de los catálogos de las EC mediante formas de acceso abierto y/o bajo licencias copyleft, sumado a la libre disponibilidad de títulos digitalizados por los usuarios a la web Scribd, nos remite también a una doble estrategia: la incorporación de criterios estéticos y poéticos por parte del lector en la construcción del catálogo general en digital y, por otro lado, el sostenimiento de la ritualidad de resistencia de las EC también en formato digital mediante la promoción de los títulos propios en acceso abierto. 
El campo editorial, así, se ha expandido a un mayor número de formas materiales, que van desde fórmulas de edición de las EC latinoamericanas hasta el formato AZW3 de Kindle (V. Figura 1). La irrupción de la dispositividad material cartonera -con su incipiente irrupción en el ecosistema digitalexpresa una mayor amplitud en las maneras de concebir la escritura y la lectura, también ligadas a las formas materiales, impresas, "tipográficas".

\section{BIBLIOGRAFÍA}

Alonso Arévalo, Julio y Marta Vázquez Vázquez. "La biblioteca como editora de contenidos". Valencia: Universidad Politécnica de Valencia. Revista Métodos de información (MEI), Vol. 6, nº 11 (2015): 201-213.

Alonso Berrocal, José Luis; Raquel Gómez Díaz; Carlos García Figuerola; Ángel Zazo Rodríguez y José Antonio Cordón García. "Propuesta de estudio del campo semántico de los libros electrónicos en Twitter”. Zaragoza: Revista Scire: representación y organización del conocimiento 17:2 (2011): 87-97 . [Consulta: 09/03/2916] Disponible en: http://ibersid. eu/ojs/index.php/scire/article/view/3939/3710

Bilbija, Ksenija. "Borrón y cuento nuevo: las EC latinoamericanas". [En línea] Caracas: Revista Nueva Sociedad, nº 230 (2010): 95-114.

Bourdieu, Pierre. Campo de poder, campo intelectual. Itinerario de un concepto. Buenos Aires: Montressor, 1966.

Celis Carbajal, Paloma. Entrevista personal, 2015.

Certeau, Michel de. La invención de lo cotidiano. Tomos I y II. México: Universidad Iberoamericana / ITESO, 1996.

Chartier, Roger. El mundo como representación. Estudios sobre historia cultural. Barcelona: Editorial Gedisa, 1992.

Inscribir y borrar. Cultura escrita y literatura (siglos XI-XVIII). Buenos Aires: Katz Editores, 2006.

Clarke, John (2014). "Estilo". En Hall, Stuart y Tony Jefferson, editores. Rituales de resistencia. Subculturas juveniles en la Gran Bretaña de Posguerra. Madrid: Traficantes de Sueños, 2014.

Cordón García, José Antonio. "La visibilidad en los circuitos de la creación: literatura y traducción". Manual de documentación y terminología para la traducción especializada. Gonzalo García, Consuelo y Valentín García Yebra, editores. Madrid: Arco/Libros (2004): $1-46$.

Lecturas sobre la lectura. Salamanca: Repositorio Gredos de la Universidad de Salamanca, 2006. [Consulta: 05/04/2016]. Disponible en: http://gredos.usal.es/jspui/ bitstream/10366/83139/1/DBD_CordonGarcia_Lecturassobrelalectura.pdf

Cordón García, José Antonio y García-Figuerola, Carlos. "Introducción. Aventuras, inventos y mixtificaciones del libro electrónico". Libros electrónicos y contenidos digitales en la sociedad del conocimiento. Mercado, servicios y derechos. José Antonio Cordón y José 
Antonio Cordón García; Fernando Carbajo Cascón; Raquel Gómez Díaz y Julio AlonsoArévalo, coordinadores. Madrid: Pirámide (2012): 19-45.

Costa, Jordi. "Notas sobre lo Trash. Apuntes para la redención de una contracultura mutante". Cultura porquería. Una espeleologia del gust. Costa, Jordi; Pedro Calleja; Rodrigo Fresán; Irwin Chusid; Sergi Pàmies; Mondo Brutto; Àlex Z. Barcelona: Centre de Cultura Contemporània de Barcelona (2003): 144-153.

Defourny Jacques. "Orígenes, contextos y funciones de un tercer gran sector". Economía Social. Entre economía capitalista y economía pública. Monzón, José Luis y Jacques Defourny, directores. Valencia: Ciriec-España (1992): 79-104.

García Canclini, Néstor. La globalización imaginada. Buenos Aires: Editorial Paidós, 1999.

García Canclini, Néstor; Francisco Cruces y Maritza Urteaga Castro Pozo. Jóvenes, culturas urbanas y redes digitales. Buenos Aires: Ariel/Fundación Telefónica, 2012.

Garza, Mercedes de la. Literatura maya. Antología. Caracas: Biblioteca Ayacucho, 1980.

Graw, Isabelle. ¿Cuánto vale el arte? Buenos Aires: Mardulce, 2013.

Hall, Suart. "The local and the Global: Globalization and Ethnicity". Culture Globalization and the World-System. Contemporary Conditions for the Representation of Identity. King, Anthony D., editor, Binghamton, Nueva York: Macmillan/State University of New York at Binghamton (1991): 19- 39.

Hall, Stuart y Tony Jefferson. Editores. Rituales de resistencia. Subculturas juveniles en la Gran Bretaña de Posguerra. Madrid: Traficantes de Sueños, 2014.

Klinger, Diana. "A arte murmurada au redor do fogo. (Um mapa possivel da narrativa latinoamericana do presente)". [En línea] Buenos Aires - Río de Janeiro: Grumo, nº 7 (2008):1219. Disponible en: http://www.salagrumo.org/download/grumo_07.pdf

Lévi-Strauss, Claude. El pensamiento salvaje. Madrid: Fondo de Cultura Económica, 1964.

Lotman, Iuri M. 1981. La semiósfera I. Semiótica de la cultura y del texto. Madrid: Cátedra, 1996.

La semiósfera II. Semiótica de la cultura, del texto, de la conducta y del espacio. Madrid: Cátedra, 1998.

Ludmer, Josefina. "Literaturas postautónomas 2.01". [En línea]. Buenos Aires: Propuesta Educativa $n^{\circ} 32$ (2009): 41-45.

Mignolo, Walter. "Los cánones y (más allá de) las fronteras culturales (o ¿de quién es el canon del que hablamos?)”. En Sullá, Enric (editor), El canon literario. Madrid: Arco- Libros, (1998): 237-270.

O’Hagan, Minako. "Multidimensional Translation: A Game Plan for Audiovisual Translation in the Age of GILT". Proceedings of the Marie Curie Euroconferences MuTra 'Challenges of Multidimensional Translation' - Saarbrücken 2-6 May 2005. Nauert, Sandra y Gerzymisch-Arbogast, Heidrun. Saarbrücken: MuTra, 2007. 76-87. Disponible en http:// translationconcepts.org/pdf/MuTra_2005_Proceedings.pdf\#page $=80$

Smith, Terry. ¿Qué es el arte contemporáneo? Buenos Aires: Siglo Veintiuno Editores, 2012.

Sotomayor, Olga. Entrevista personal, 2016. 
University of Wisconsin Digital Collections. Base de Datos de Editoriales Cartoneras. [Web] Madison, Wisconsin: Universidad de Wisconsin.[Consulta: 05/12/2015] Disponible en: http://digicoll.library.wisc.edu/WebZ/SearchOrBrowse?sessionid=01-53965-684142241

Vila, Adrián. El canon oculto. La literatura de América Latina y el Caribe en la transposición al ecosistema digital. Salamanca: Ediciones Universidad de Salamanca, 2016.

Wischenbart, Rüdiger; Carlo Carrenho; Veronika Licher; Miha Kovac y Mallya Vinhuta. Global eBook: Current Conditions \& Future Projections. Londres: O’Reillym, 2013. 